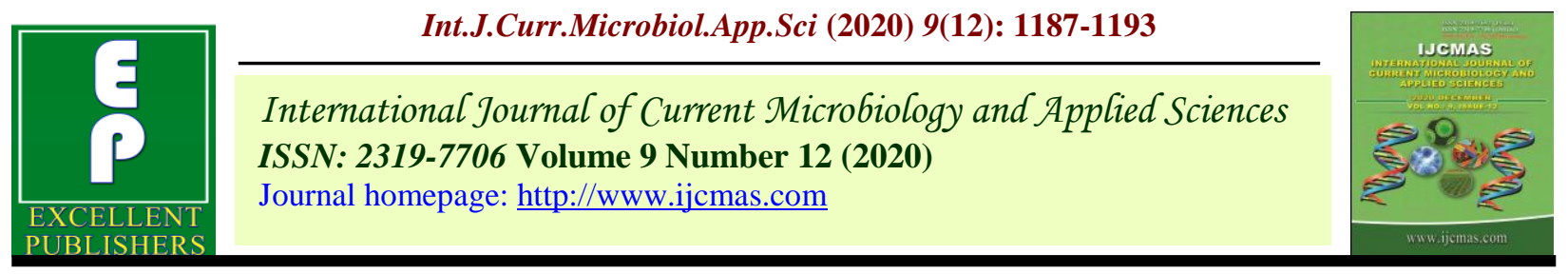

Original Research Article

https://doi.org/10.20546/ijcmas.2020.912.145

\title{
Economic Impact of Farm Ponds on Beneficiary and Non-beneficiary Farmers for Tur (Arhar) Cultivation in Akola Tahasil
}

\author{
Rushikesh K. Patil*, A.S. Tingre, Jayshri R. Kankate and Ankita D. Chakranarayan
}

Department of Agriculture Economics and Statistics, Post Graduate Institute, Akola, Maharashtra, India

*Corresponding author

Keywords

Farm pond,

Productivity, Cost

A, Cost B, Cost C,

Akola, Output-input ratio

Article Info

Accepted:

10 November 2020

Available Online:

10 December 2020

\begin{abstract}
A B S T R A C T
The present study entitled Comparative economics of farm ponds beneficiary and non-beneficiary farmers in Akola tahasil. A farm pond is a large hole dug out in the earth, usually square or rectangular in shape, which harvests rainwater and stores it for future use. Economic evaluation of farm pond is necessary for the effective implementation. The main objective of present study was to assess the impact of farm ponds on productivity of various inputs used by farmers. This study was undertaken in Akola tahasil. The study was based on a sample of 60 beneficiary and 60 non-beneficiary farmers data pertaining the year 2017-18 were collected by survey method from the beneficiary and non-beneficiary farmers. The sample farmers were personally contacted and primary data was collected from them in a specially structured schedule i.e., cost ' $A$ ', cost ' $\mathrm{B}$ ' and cost ' $\mathrm{C}$ ' was used for the analysis of data. It is observed from the study that output-input ratio of beneficiary farmers was higher as compare to non-beneficiary farmers. It shows that the beneficiary farmers were more profitable than non-beneficiary farmers.
\end{abstract}

\section{Introduction}

In recent year India has looked to farm pond as a way to realize its hopes for agricultural development in rainfed, semi-arid areas. These areas were bypassed by the Green Revolution and have experienced little or no growth in agricultural production for several decades. By capturing scarce water resources and improving the management of soil and vegetation, farm pond development has the potential to create conditions conducive to higher agricultural productivity, while conserving natural resources.

Farm pond activity is an adaptive, comprehensive and constructive technique for multi-resource management that seek to balance healthy, economic and social conditions within a watershed. Farm pond serves to integrate planning regarding better utilization of land and water; taking in account to both groundwater as well as surface water flow, so as to create or 
recognize new and sustainable methods for the interaction of water, plants, animals and human land use found within the physical boundaries of a farm pond.

Farm pond activity basically refers to efficient management and conservation of surface and groundwater resources. It involves prevention of runoff and storage and recharge of groundwater through various methods like percolation tanks, recharge wells, etc. However, in broad sense farm pond management includes conservation, regeneration and judicious use of all resources - natural (like land, water, plants and animals) and human with in a farm pond. Farm pond management aims at bringing about balance between natural resources on the one hand and society on the other. The success of farm pond development largely depends upon community participation. Farm pond management as the tool for sustainable development.

Agriculture is the backbone of Indian economy and society. In Vidarbha region, farmer suicide is serious and current issue. Due to unfavourable climatic and natural conditions such as low rainfall, drought, hailstorms etc., it is difficult to farmers to withstand in such conditions. Day by day percentage of rainfall is decreasing. Groundwater availability is getting very low and available water resources are not fulfil need of farmers. So now a days to create the awareness among the people about farm pond management is very necessary. Farm pond management is done with help of check dams, percolation ponds, stone barriers, slopping lands, bench terracing, etc. In this works, village community play vital role and employment for villagers is also generated.

\section{Materials and Methods}

The present study entitled comparative economics of farm ponds beneficiary and non-beneficiary farmers were be conducted for Akola tahasil.

\section{Economics of production}

For working out the economics of production of the crops, the costs of cultivation were worked out .i.e. cost A, cost B and cost C. The various items included in the cost of cultivation and the methods adopted in their evaluation are presented in the following section.

\section{Human labour}

While estimating the cost of human labour, both hired and family labours were taken into consideration. For estimating the cost of hired labour, wages actually paid to the labours were taken to consideration. Family labour was evaluated at the cost of wages paid to the hired labour.

\section{Bullock labour}

The appropriate method to estimate the cost of bullock labour is to estimate the net costs of maintenance of bullock pair and divide it by the total numbers of work days of the bullock pair. This method, however, requires more data and time for its estimation. In the alternative, therefore, the bullock labour was valued on the basis of the prevailing rate of bullock pair in the locality for various operations.

\section{Seed}

The seeds purchased from the market were evaluated on the basis of price actually paid. The farm produced seeds were evaluated on the basis of market price.

\section{Manure}

The manure purchased from outside was evaluated at the price actually paid. While the 
manure produced on farm was evaluated at the price prevailing in the village.

\section{Fertilizers}

The actual cost incurred on the purchase of fertilizer was taken into consideration.

\section{Pesticides}

The actual expenditure incurred on the purchase of pesticide was taken into consideration.

\section{Irrigation charge}

Irrigation charges included the actual energy bill, depreciation on pump set and maintenance of irrigation machinery. Total irrigation charges were distributed among irrigation crops on the basis of area.

\section{Return}

The total return from a crop comprise of value of main produce and by produce. For calculating the value of main product, the harvest price of the crop was considered. In case of by produce the prevailing price in the locality was considered for calculating its value. The total returns from a crop were obtained by multiplying the quantity of main and by product by their respective per unit prices and added together to get total value.

\section{Results and Discussion}

The findings of the present study as well as relevant discussion have been presented under following heads:

\section{Per hectare input utilization of Tur (Arhar)}

Result presented in table 1 indicates that at overall level the use of various inputs for tur on beneficiary farm was slightly higher than non-beneficiary farms. Beneficiary farmers used 32.79 hired male labour days per hectare as against 31.52 male labours days by nonbeneficiary farmers. Use of hired female labour days was slightly different on beneficiary farms i.e. 59.15 days than nonbeneficiary farms i.e. 58.33 days. Use of seed and farm yard manure per hectare did not vary much on beneficiary farms and nonbeneficiary farms. Use of chemical fertilizers on beneficiary farms $(129.11 \mathrm{~kg}$.) was slightly higher than non-beneficiary farms (126.78 $\mathrm{kg}$.). Intergroup comparison of beneficiary and non-beneficiary farms also revealed the same trend.

\section{Per hectare cost of cultivation of Tur (Arhar) for beneficiary and non- beneficiary farmers}

Results on the economics of production of tur are presented in table 2 . It could be seen from the table that at the overall level beneficiary farmers incurred an expenditure of Rs. 88, 330.56 per hectare in the cultivation of tur. Human labour was the main item of expenditure accounting for about 20.44 per cent hired and 10.34 per cent family labour of the total cost. Bullock labour expenditure accounting for 5.38 per cent of total cost. Manures and fertilizers together accounted for about 6.11 per cent of the total cost. Expenditure on plant protection was practically 1.54 per cent. Intergroup comparison revealed that the highest expenditure of Rs. 97,451.66 was incurred by large farmers. Small and medium farmers incurred an amount of Rs. 81,386.94 and Rs. $86,153.09$ in the cultivation of tur.

In non-beneficiary group per hectare expenditure in the cultivation of tur at overall level was Rs. 78,292.49. The important items of expenditure in items of their share in their total cost were human labour (22.45 per cent 
hired and 11.32 per cent family labour), bullock labour (5.33 per cent) and manures and fertilizers (6.76 per cent). As in case of beneficiary farmers, highest expenditure in cultivation of tur was incurred by large farmers in non-beneficiary group also i.e. Rs. 87,342.27.

Beneficiary farmers, on an average, obtained yield of 22.41 quintals per hectare, while non- beneficiary farmers obtained 18.62 quintals yield per hectare. Thus beneficiary farmers obtained about 3.79 quintals higher yield per hectare than the non-beneficiary farmers. Intergroup comparison revealed that beneficiary farmers of all the size groups obtained higher yield than their counter-parts in the non-beneficiary group.

Table.1 Per hectare input utilization for Tur (Arhar) for beneficiary and non-beneficiary farmers

\begin{tabular}{|c|c|c|c|c|c|c|c|c|c|c|}
\hline \multirow{3}{*}{$\begin{array}{l}\text { Sr. } \\
\text { No }\end{array}$} & \multirow[t]{3}{*}{ Particulars } & \multirow[t]{3}{*}{ Unit } & \multicolumn{8}{|c|}{ Land holding size } \\
\hline & & & \multicolumn{2}{|c|}{ Small } & \multicolumn{2}{|c|}{ Medium } & \multicolumn{2}{|c|}{ Large } & \multicolumn{2}{|c|}{ Overall } \\
\hline & & & B & NB & B & NB & B & NB & B & NB \\
\hline \multirow[t]{4}{*}{1} & $\begin{array}{l}\text { Hired } \\
\text { labour }\end{array}$ & & & & & & & & & \\
\hline & i) Male & Days & 30.15 & 29.10 & 32.82 & 31.32 & 35.41 & 34.14 & 32.79 & 31.52 \\
\hline & ii)Female & Days & 60.59 & 60.23 & 58.45 & 57.56 & 58.41 & 57.19 & 59.15 & 58.33 \\
\hline & Total & & 90.74 & 89.33 & 91.27 & 88.88 & 93.82 & 91.33 & 91.94 & 89.85 \\
\hline \multirow[t]{4}{*}{2} & $\begin{array}{l}\text { Family } \\
\text { labour }\end{array}$ & & & & & & & & & \\
\hline & i) Male & Days & 30.12 & 29.16 & 32.14 & 32.58 & 31.97 & 30.51 & 31.41 & 30.75 \\
\hline & ii) Female & Days & 16.15 & 14.95 & 14.27 & 13.87 & 13.80 & 12.81 & 14.74 & 13.88 \\
\hline & Total & & 46.27 & 44.11 & 46.41 & 46.45 & 45.77 & 43.32 & 46.15 & 44.63 \\
\hline 3 & $\begin{array}{l}\text { Bullock } \\
\text { labour }\end{array}$ & $\begin{array}{l}\text { Pair } \\
\text { days }\end{array}$ & 8.81 & 7.41 & 7.30 & 7.03 & 7.67 & 6.42 & 7.93 & 6.95 \\
\hline 4 & $\begin{array}{l}\text { Machine } \\
\text { labour }\end{array}$ & Hours & 79.45 & 78.54 & 81.64 & 80.54 & 89.78 & 88.56 & 83.62 & 82.55 \\
\hline 5 & Seed & $\mathrm{Kg}$ & 29.02 & 28.15 & 29.56 & 28.41 & 29.94 & 28.84 & 29.51 & 28.47 \\
\hline 6 & Manure & Qtls & 53.04 & 52.25 & 54.21 & 52.65 & 55.41 & 53.68 & 54.22 & 52.86 \\
\hline 7 & Irrigation & No. & 3.06 & 0.00 & 3.46 & 0.00 & 3.87 & 0.00 & 3.46 & 0.00 \\
\hline \multirow[t]{5}{*}{8} & $\begin{array}{l}\text { Fertilizers } \\
\text { (NPK) }\end{array}$ & & & & & & & & & \\
\hline & $\mathrm{N}$ & $\mathrm{Kg}$ & 63.41 & 62.12 & 64.89 & 63.98 & 66.12 & 65.23 & 64.81 & 63.78 \\
\hline & $\mathrm{P}$ & $\mathrm{Kg}$ & 45.78 & 44.65 & 46.24 & 45.67 & 48.06 & 47.85 & 46.69 & 46.06 \\
\hline & $\mathrm{K}$ & $\mathrm{Kg}$ & 16.54 & 16.32 & 17.87 & 17.00 & 18.42 & 17.53 & 17.61 & 16.95 \\
\hline & Total & $\mathrm{Kg}$ & 125.73 & 123.09 & 129.00 & 126.65 & 132.60 & 130.61 & 129.11 & 126.78 \\
\hline 9 & $\begin{array}{l}\text { Plant } \\
\text { protection }\end{array}$ & Rs. & 5543.12 & 5426.45 & 5745.73 & 5564.23 & 5841.46 & 5784.86 & 5710.10 & 5591.85 \\
\hline
\end{tabular}

Note: (Figures in parentheses indicates the percentages over cost ' $\mathrm{C}$ ') $\mathrm{B}=$ Beneficiary, NB=Non beneficiary 
Table.2 Per hectare cost of cultivation of Tur (Arhar) for beneficiary and non-beneficiary farmers

\begin{tabular}{|c|c|c|c|c|c|c|c|c|c|}
\hline \multirow{3}{*}{$\begin{array}{l}\text { Sr. } \\
\text { No. }\end{array}$} & \multirow[t]{3}{*}{ Particulars } & \multicolumn{6}{|c|}{ Land holding size } & \\
\hline & & \multicolumn{2}{|c|}{ Small } & \multicolumn{2}{|c|}{ Medium } & \multicolumn{2}{|c|}{ Large } & \multicolumn{2}{|c|}{ Overall } \\
\hline & & B & NB & B & NB & B & NB & B & NB \\
\hline \multirow[t]{7}{*}{1} & \multicolumn{9}{|l|}{ Hired labour } \\
\hline & \multirow[t]{2}{*}{ i) Male } & 8442.00 & 8148.00 & 9189.60 & 8769.60 & 9914.80 & 9559.20 & 182.13 & 8825.60 \\
\hline & & $(6.81)$ & $(7.87)$ & $(6.46)$ & $(7.22)$ & (4.96) & $(5.59)$ & $(5.99)$ & $(6.77)$ \\
\hline & \multirow{2}{*}{ ii)Female } & 9088.50 & 9034.50 & 8767.50 & 8634.00 & 8761.50 & 8578.50 & 8872.50 & 8749.00 \\
\hline & & (11.17) & $(12.87)$ & $(10.18)$ & (11.17) & (8.99) & $(9.82)$ & (10.04) & (11.17) \\
\hline & \multirow[t]{2}{*}{ Total } & 17530.50 & 17182.50 & 17957.10 & 17403.60 & 18676.30 & 18137.70 & 18054.63 & 17574.60 \\
\hline & & (21.54) & $(24.47)$ & (20.84) & $(22.51)$ & (19.16) & (20.77) & (20.44) & $(22.45)$ \\
\hline \multirow[t]{2}{*}{2} & \multirow[t]{2}{*}{ Bullock lab } & 5286.00 & 4446.00 & 4380.00 & 4218.00 & 4602.00 & 3852.00 & 4756.00 & 4172.00 \\
\hline & & $(6.49)$ & $(6.33)$ & $(5.08)$ & $(5.46)$ & $(4.72)$ & $(4.41)$ & $(5.38)$ & $(5.33)$ \\
\hline \multirow[t]{2}{*}{3} & \multirow{2}{*}{$\begin{array}{l}\text { Machine } \\
\text { charges }\end{array}$} & 11917.50 & 11781.00 & 12246.00 & 12081.00 & 13467.00 & 13284.00 & 12543.50 & 12382.00 \\
\hline & & (14.64) & $(16.78)$ & $(14.21)$ & $(15$ & $(13.82)$ & $(15$ & $(14.20)$ & 2) \\
\hline \multirow[t]{2}{*}{4} & \multirow[t]{2}{*}{ Seed } & 464 & 45 & 4729.60 & 45 & 4790.40 & & 4721.07 & 4.67 \\
\hline & & $(5.71)$ & $(6.41)$ & 49) & $(5.8$ & $(4.92)$ & $(5.2)$ & $(5.34)$ & 2) \\
\hline \multirow[t]{2}{*}{5} & \multirow{2}{*}{ Manure } & 803 & 77 & 74 & 797 & 838 & 2 & .89 & 30 \\
\hline & & $(0.9$ & 3) & 5) & $(1.0$ & $(0$ & & 3) & 2) \\
\hline \multirow{2}{*}{6} & \multirow{2}{*}{ Irrigation } & 167 & 0.00 & 25 & .00 & 14 & 0 & .11 & \\
\hline & & $(1)$. & 00 & 5) & 0.00 & ) & 0.0 & 0) & 0.00 \\
\hline \multirow{2}{*}{7} & \multirow{2}{*}{ Fertilizers } & 446 & & & & 46 & 4639.48 & 45 & 4495.04 \\
\hline & & $(5.4$ & 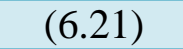 & $(5$ & ( & & $(5$ & 8) & $(5.74)$ \\
\hline \multirow{2}{*}{8} & \multirow{2}{*}{ Plant protection } & 1456 & 156 & 1348.54 & 148 & 1285.49 & 134 & 1363.52 & 1466.07 \\
\hline & & $(1$. & & & 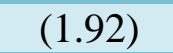 & & & 4) & $(1.87)$ \\
\hline 9 & Incidental & 774 & 760 & 84 & 8 & 94 & 987 & 54.84 & 877.98 \\
\hline & & $(0$. & ( & 3) & & 7) & $(1$. & $(0.97)$ & $(1.12)$ \\
\hline 10 & Repairing & 671.22 & 65 & 76 & 712 & 880.46 & 79 & 771.51 & 722.74 \\
\hline & & $(0.8$ & $(0.9$ & $(0.89)$ & $(0.9$ & $(0.90)$ & $(0$. & 7) & $0.92)$ \\
\hline & Working capital & 49219.24 & 46049.64 & 49483.48 & 46612.10 & 52294.96 & 48474.47 & 50332.56 & 47045.40 \\
\hline & & $(60$ & $(56$. & $(60$ & $(5$ & $(63.97)$ & $(55$ & 61) & $(57.80)$ \\
\hline 11 & $\begin{array}{l}\text { Interest on } \\
\text { working capital } \\
@ 6 \% \text { per } \\
\text { annum for crop } \\
\text { period }\end{array}$ & $\begin{array}{c}1476.58 \\
(1.81)\end{array}$ & $\begin{array}{c}1381.49 \\
(1.97)\end{array}$ & $\begin{array}{c}1484.50 \\
(1.72)\end{array}$ & $\begin{array}{c}1398.36 \\
(1.81)\end{array}$ & $\begin{array}{c}1568.85 \\
(1.60)\end{array}$ & $\begin{array}{c}1454.23 \\
(1.66)\end{array}$ & $\begin{array}{c}1509.98 \\
(1.70)\end{array}$ & $\begin{array}{c}1411.36 \\
(1.80)\end{array}$ \\
\hline 12 & $\begin{array}{l}\text { Depreciation } \\
\text { cost }\end{array}$ & $\begin{array}{c}5470.40 \\
(6.72)\end{array}$ & $\begin{array}{l}2257.95 \\
(3.22)\end{array}$ & $\begin{array}{c}7130.50 \\
(8.28)\end{array}$ & $\begin{array}{c}4987.35 \\
(6.45)\end{array}$ & $\begin{array}{c}10886.60 \\
(11.17)\end{array}$ & $\begin{array}{l}8541.80 \\
(9.78)\end{array}$ & $\begin{array}{c}7829.17 \\
(8.86)\end{array}$ & $\begin{array}{c}5262.37 \\
(6.72)\end{array}$ \\
\hline 13 & $\begin{array}{l}\text { Land revenue } \\
\text { and other taxes }\end{array}$ & $\begin{array}{l}12.20 \\
(0.01)\end{array}$ & $\begin{array}{l}11.25 \\
(0.02)\end{array}$ & $\begin{array}{l}26.44 \\
(0.03)\end{array}$ & $\begin{array}{l}24.95 \\
(0.03)\end{array}$ & $\begin{array}{l}55.87 \\
(0.06)\end{array}$ & $\begin{array}{l}52.34 \\
(0.06)\end{array}$ & $\begin{array}{l}31.50 \\
(0.04)\end{array}$ & $\begin{array}{l}29.52 \\
(0.04)\end{array}$ \\
\hline & Cost ' $A$ ' & $\begin{array}{c}56178.42 \\
(68.79)\end{array}$ & $\begin{array}{c}49700.33 \\
(70.78)\end{array}$ & $\begin{array}{c}58124.93 \\
(67.28)\end{array}$ & $\begin{array}{c}53022.76 \\
(68.58)\end{array}$ & $\begin{array}{c}64806.27 \\
(66.25)\end{array}$ & $\begin{array}{c}58522.84 \\
(67.00)\end{array}$ & $\begin{array}{c}59703.21 \\
(67.37)\end{array}$ & $\begin{array}{c}53748.64 \\
(68.65)\end{array}$ \\
\hline
\end{tabular}




\begin{tabular}{|c|c|c|c|c|c|c|c|c|c|}
\hline 14 & $\begin{array}{l}\text { Interest on fixed } \\
\text { capital @ 10\% }\end{array}$ & $\begin{array}{c}4815.86 \\
(5.92)\end{array}$ & $\begin{array}{c}2710.59 \\
(3.86)\end{array}$ & $\begin{array}{c}5851.75 \\
(6.79)\end{array}$ & $\begin{array}{c}4087.26 \\
(5.29)\end{array}$ & $\begin{array}{c}7792.01 \\
(8.00)\end{array}$ & $\begin{array}{c}6171.12 \\
(7.07)\end{array}$ & $\begin{array}{c}6153.21 \\
(6.97)\end{array}$ & $\begin{array}{c}4322.99 \\
(5.52)\end{array}$ \\
\hline \multirow[t]{2}{*}{15} & $\begin{array}{l}\text { Rental value of } \\
\text { land }\end{array}$ & $\begin{array}{c}11549.80 \\
(14.19)\end{array}$ & $\begin{array}{l}9152.25 \\
(13.03)\end{array}$ & $\begin{array}{c}13107.22 \\
(15.21)\end{array}$ & $\begin{array}{c}10935.72 \\
(14.14)\end{array}$ & $\begin{array}{c}15968.30 \\
(16.39)\end{array}$ & $\begin{array}{c}13990.16 \\
(16.02)\end{array}$ & $\begin{array}{c}13541.77 \\
(15.33)\end{array}$ & $\begin{array}{c}11359.37 \\
(14.51)\end{array}$ \\
\hline & Cost 'B' & $\begin{array}{c}72348.94 \\
(88.90)\end{array}$ & $\begin{array}{c}61563.17 \\
(87.68)\end{array}$ & $\begin{array}{c}76923.79 \\
(89.29)\end{array}$ & $\begin{array}{c}68045.73 \\
(88.01)\end{array}$ & $\begin{array}{c}88326.41 \\
(90.64)\end{array}$ & $\begin{array}{c}78684.12 \\
(90.09)\end{array}$ & $\begin{array}{c}79199.71 \\
(89.66)\end{array}$ & $\begin{array}{c}69431.01 \\
(88.68)\end{array}$ \\
\hline \multicolumn{10}{|c|}{16 Family labour } \\
\hline & i) Male & $\begin{array}{c}6777.00 \\
(8.33)\end{array}$ & $\begin{array}{l}6561.00 \\
(9.34)\end{array}$ & $\begin{array}{c}7231.50 \\
(8.39)\end{array}$ & $\begin{array}{c}7330.50 \\
(9.48)\end{array}$ & $\begin{array}{l}7193.25 \\
(7.38)\end{array}$ & $\begin{array}{c}6864.75 \\
(7.86)\end{array}$ & $\begin{array}{c}7067.25 \\
(8.00)\end{array}$ & $\begin{array}{l}6918.75 \\
(8.84)\end{array}$ \\
\hline & ii) Female & $\begin{array}{c}2261.00 \\
(2.78)\end{array}$ & $\begin{array}{c}2093.00 \\
(2.98)\end{array}$ & $\begin{array}{c}1997.80 \\
(2.32)\end{array}$ & $\begin{array}{c}1941.80 \\
(2.51)\end{array}$ & $\begin{array}{c}1932.00 \\
(1.98)\end{array}$ & $\begin{array}{c}1793.40 \\
(2.05)\end{array}$ & $\begin{array}{c}2063.60 \\
(2.34)\end{array}$ & $\begin{array}{c}1942.73 \\
(2.48)\end{array}$ \\
\hline & Total & $\begin{array}{c}9038.00 \\
(11.10)\end{array}$ & $\begin{array}{l}8654.00 \\
(12.32)\end{array}$ & $\begin{array}{l}9229.30 \\
(10.71)\end{array}$ & $\begin{array}{l}9272.30 \\
(11.99)\end{array}$ & $\begin{array}{c}9125.25 \\
(9.36)\end{array}$ & $\begin{array}{c}8658.15 \\
(9.91)\end{array}$ & $\begin{array}{l}9130.85 \\
(10.34)\end{array}$ & $\begin{array}{l}8861.48 \\
(11.32)\end{array}$ \\
\hline & Cost ' C' & $\begin{array}{c}81386.94 \\
(100.00)\end{array}$ & $\begin{array}{c}70217.17 \\
(100.00)\end{array}$ & $\begin{array}{c}86153.09 \\
(100.00)\end{array}$ & $\begin{array}{c}77318.03 \\
(100.00)\end{array}$ & $\begin{array}{c}97451.66 \\
(100.00)\end{array}$ & $\begin{array}{c}87342.27 \\
(100.00)\end{array}$ & $\begin{array}{c}88330.56 \\
(100.00)\end{array}$ & $\begin{array}{c}78292.49 \\
(100.00)\end{array}$ \\
\hline \multicolumn{10}{|c|}{17 Output (Qtls.) } \\
\hline & i) Main produce & 19.68 & 16.22 & 21.58 & 17.25 & 25.97 & 22.39 & 22.41 & 18.62 \\
\hline & ii) By produce & 8.41 & 6.58 & 9.89 & 8.15 & 13.45 & 11.58 & 10.58 & 8.77 \\
\hline 18 & $\begin{array}{l}\text { Gross Return } \\
\text { (Rs) }\end{array}$ & 73371.00 & 60366.00 & 80655.00 & 64545.00 & 97527.00 & 84078.00 & 88438.83 & 73474.70 \\
\hline 19 & $\begin{array}{l}\text { Output input } \\
\text { ratio at cost ' } A \text { ' }\end{array}$ & 1.31 & 1.21 & 1.39 & 1.22 & 1.50 & 1.44 & 1.48 & 1.37 \\
\hline
\end{tabular}

Note: (Figures in parentheses indicates the percentages over cost ' $C$ ') $B=B e n e f i c i a r y, ~ N B=N o n$

A perusal of table 5.3.3 reveals that there was not much difference in the level of inputs use among the beneficiary and non-beneficiary farmers. In spite of this per hectare yield of tur on beneficiary farms was higher than the non-beneficiary farms. Gross returns per hectare from tur cultivation on beneficiary farms was Rs 88,438.38. Gross return varied from Rs. 73,371 in small group to Rs. 80,655 in medium group and Rs. 97,527 in large group. In non-beneficiary group gross returns per hectare at overall level was Rs. $73,474.70$ and it varied from Rs. 60,366 in small group to Rs. 64,545 in medium group and Rs. 84,078 in large group. Gross returns per hectare from cultivation of tur were higher in all the size group of beneficiary farms than the non-beneficiary farms. This was obviously due to higher per hectare yield of tur on beneficiary farms.

Comparison of output input ratios over cost
'A' at overall level shows that beneficiary farmers (1.48) obtained higher returns over investment than the non-beneficiary farmers (1.37) from the cultivation of tur.

In fertilizers and manures was used more in case of beneficiary farmers as compared to non-beneficiary farmers while bullock labour, seed, machinery was more in case nonbeneficiary farmers as compared to beneficiary conclusions, at overall level family labour, farmers.

At overall level per hectare cost of cultivation i.e. cost $\mathrm{C}$ for tur crop in case of nonbeneficiary farmers was Rs. 78,292.49 which was less than cost of Rs. 88,330.56 in case of beneficiary farmers.

In case of beneficiary farms input output ratio on cost A at overall level for tur crop was 
1.48 which was higher than non-beneficiary farms with input output ratio of 1.37 .

The impact of farm pond construction on their field increase water level of well and also for their field to provide water during crop season whenever necessary to increase a crop production. Higher regression coefficient and consequently higher factor productivity for beneficiary farms were obviously due to farm pond availability on these farms

\section{References}

1. Daulat Singh, 1971. Economics of soybean cultivation. Rural India. 34(4): 78.

2. Gangwar, A.C. and R.N. Pandey. 1982. Stagnation in production of pulses: an economic analysis, Agril. Situation in India 37(5):282-287.

3. Garg, K.P. 1985. Economics of production of soybean. Dryland agriculture. 259-261.

4. Gadre, N.P. and Y.P. Mahalle.1988. Comparative economics of production of cotton in Vidarbha region. Indian $\mathrm{J}$. Agril. Econ. 43(2): 514.

5. Gondhali, R.S., Ulemale, D.H. and Sarap, S.M. 2017. Economics analysis of Gram in Amravati district. Internat. Res. J. Agric. Eco. \& Stat., 8(1): 31-36.

6. Kumar Singh, M.S. 1996. Economics of production of cotton vis-a-vis soybean followed by rabi crop in selected area of Nagpur district under rainfed condition. M.Sc. Thesis (Unpub.): 63.

7. Singh, J. P. and H. N. Attibudhi. 1985. Economics of pulses cultivation in Cuttack Sadar of Cuttack district. Orrisa Agril. Situation in India. 40(8): 514.

8. Singh, R. and S. M. A. Rizvi .1988. Comparative economics of production of soybean and it's competing crops in Uttar Pradesh Indian J. Agril. Econ. 43(2): 514.

9. Surykant, P. N. Singh, S. Kuthe and N.R. Medat. 2016. Input Use, Costs Structure and Return Analysis of Soybean in South Gujarat. International Journal of Agriculture Sciences, Volume 8, Issue 52, pp.-2508-2510.

10. Visawadia, H.R.; Fadadu, A.M. and Tarpara, V.D. 2006. A comparative analysis of production and marketing of Bt. Cotton and Hybrid cotton in Saurashtra region of Gujarat State. Agril. Econ. Res. Review. 19: 293-300.

11. Verma, A.R., A. M. Rajput and L. R. Kashyap. 2002. Economics of production in Khargaon district of Madhya Pradesh. Ind. J. Agril. Mktg. 16 (2): 89-97.

12. Wagh, H.J., Lamtule, J.A. and Kadam, M.M. 2014. Input utilization in tur production - A production function analysis. Internat. Res. J. Agric. Eco. \& Stat., 5(2): 253-256.

\section{How to cite this article:}

Rushikesh K. Patil, A.S. Tingre, Jayshri R. Kankate and Ankita D. Chakranarayan. 2020. Economic Impact of Farm Ponds on Beneficiary and Non-beneficiary Farmers for Tur (Arhar) Cultivation in Akola Tahasil. Int.J.Curr.Microbiol.App.Sci. 9(12): 1187-1193. doi: https://doi.org/10.20546/ijcmas.2020.912.145 